\title{
Grains in Astronomy - An Overview
}

\author{
Adolf N. Witt \\ Ritter Astrophysical Research Center, The University of Toledo, Toledo, \\ $\mathrm{OH}$ 43606, USA
}

\begin{abstract}
Astrophysical processes in which grains play key roles are briefly reviewed.
\end{abstract}

\section{Introduction}

Grains represent the largest known depository of solid-phase matter in the Universe, amounting to about $10^{-3}$ of the mass of galaxies during their active starforming lifetime (Edmunds \& Eales 1998). Produced initially in the outflows from mass-losing or exploding stars, grains are subsequently processed in circumstellar and interstellar environments (Jones 2000). They are found everywhere where conditions permit the existence of solids, from circumstellar outflows (Sedlmayr 1997; Sedlmayr \& Krueger 1997), to the diffuse interstellar medium of galaxies and molecular clouds (Mathis 1990), to interplanetary space (Kelsall et al. 1998), from the halos and tails of comets (Combi 1994) to the halos of galaxies (Zaritsky 1994; Alton et al. 2000). While initially considered mainly a nuisance responsible for causing continuous opacity in interstellar space, grains are now recognized as key agents in a large number of astrophysical processes. The size distribution of grains varies somewhat with the environment where they are found, depending on the balance between grain production and destruction processes (Tielens 1998), but generally extends from nanometer-sized clusters barely larger than large molecules (Witt 2000) to micrometer-sized grains (Frisch et al. 1999).

\section{Grains as Opacity Source}

Longward of the wavelength of $0.0912 \mu \mathrm{m}$ grains are the dominant source of opacity in interstellar space. They yield a cross section per atom of interstellar hydrogen of $10^{-22}$ to $10^{-21} \mathrm{~cm}^{2}$ in the wavelength range extending from the near-infrared to the far-ultraviolet, largely through scattering (Calzetti et al. 1995). Laor \& Draine (1993) provide useful data on the dust opacity for the wavelength range from 0.0001 to $1000 \mu \mathrm{m}$. Internal extinction within galaxies leads to wavelength-dependent attenuation of the UV-optical flux measurable by outside observers. This attenuation depends sensitively upon the structure of the dust distribution, its geometry with respect to the dominant sources, and the type of dust involved (Witt \& Gordon 2000). This attenuation must be modeled correctly, if the star-formation history of the early Universe is to be 
deduced from observations of the UV-optical luminosity density of galaxies at high redshift (Steidel et al. 1999; Calzetti et al. 2000).

\section{Grains as Sources of Radiation}

Most of the photon energy absorbed by grains in the UV-optical portions of the spectrum reappears in the form of dust emission at wavelengths ranging from the optical to the sub-mm (Verter et al. 2000). At least three distinct grain components appear to be necessary to explain the complex dust emission spectrum: large, sub-micron grains radiating in the far-infrared in thermal equilibrium with the radiation field; very small $\mathrm{nm}$-sized grains heated stochastically by individual photons, radiating continuum radiation over a wider range of infrared wavelengths; and very small hydrocarbon clusters responsible for the ubiquitous unidentified infrared (UIR) bands. In addition, widespread optical photoluminescence by interstellar grains is observed in many dusty astronomical environments, including the diffuse ISM at high galactic latitudes (Gordon et al. 1998). The most likely source is one of the very small grain components already mentioned above (e.g. Witt et al. 1998). Very small spinning grains also appear to be the source of microwave emission in the $10-100 \mathrm{GHz}$ frequency range (Draine \& Lazarian 1998), adding potential confusion to the cosmic microwave background radiation.

\section{Grains as Depository of Heavy Elements}

Grains represent the selective condensation of heavy elements into solids, but the degree to which this condensation leads to depletion of the elements from the gas phase differs greatly from element to element and from one astronomical environment to another (Jones 2000). Adding uncertainty to the balance between the amounts of specific elements thought to be present in grains and the amounts still observed to be in the gas phase is the fact that the so-called reference abundances, i.e. the relative abundances expected if all matter were in gas form, are quite uncertain (Snow \& Witt 1996). Through mechanisms which preferentially remove grains from galaxies (Barsella et al. 1989; Ferrara et al. $1990,1991)$, grains also play a major role in enriching galactic halo gas and intracluster gas with heavy elements, while at the same time removing heavy elements from the star-forming regions of stellar systems.

\section{Grains as Catalysts}

Grains contribute to interstellar chemistry in multiple ways. Efficient formation of molecular hydrogen, the most abundant interstellar molecule and starting point for subsequent gas-phase reactions, can occur only on the surfaces of grains (Hollenbach \& Salpeter 1971; Hollenbach et al. 1971). More complex molecules also form on grain surfaces or in ice mantles as a result of photolysis (Grim et al. 1989). Finally, the shielding against the interstellar radiation field provided by extinction through grains allows the products of gas-phase chemistry to persist and build up to observable levels in molecular clouds. In another major catalytic 
role, radiation-pressure-driven grains in the atmospheres of late-type giant stars facilitate the mass loss from such objects (Hoefner et al. 1995). Finally, via the photoelectric effect on grains, stellar photons are able to contribute to the heating of the neutral interstellar gas in the diffuse interstellar medium of galaxies (Bakes \& Tielens 1994).

\section{References}

Alton, P.B., et al. 2000, A\&AS, 145, 83

Bakes, E.L.O., \& Tielens, A.G.G.M. 1994, ApJ, 427, 822

Barsella, B., Ferrini, F., Greenberg, J.M., \& Aiello, S. 1989, A\&A, 209, 349

Calzetti, D., et al. 1995, ApJ, 446, L97

Calzetti, D., et al. 2000, ApJ, 533, 682

Combi, M.R., 1994, AJ, 108, 304

Draine, B.T., \& Lazarian, A. 1998, ApJ, 508, 157

Edmunds, M.G., \& Eales, S.A. 1998, MNRAS, 299, L29

Ferrara, A., Aiello, S., Ferrini, F., \& Bersella, B. 1990, A\&A, 240, 259

Ferrara, A., Ferrini, F., Barsella, B., \& Franco, J. 1991, ApJ, 381, 137

Frisch, P.C., et al. 1999, ApJ, 525, 492

Gordon, K.D., Witt, A.N., \& Friedmann, B.C. 1998, ApJ, 498, 522

Grim, R.J.A., et al. 1989, A\&AS, 78, 161

Hoefner, S., Feuchtinger, M.U., \& Dorfi, E.A. 1995, A\&A, 297, 815

Hollenbach, D., \& Salpeter, E.E. 1971, ApJ, 163, 155

Hollenbach, D., Werner, M.W., \& Salpeter, E.E. 1971, ApJ, 163, 165

Jones, A.P. 2000, JGR, 105, 10257

Kelsall, T. et al. 1998, ApJ, 508, 44

Laor, A., \& Draine, B.T. 1993, ApJ, 402, 441

Mathis, J.S. 1990, ARAA, 28, 37

Snow, T.P., \& Witt, A.N., 1996, ApJ, 468, L65

Sedlmayr, E. 1997, ApSS, 251, 103

Sedlmayr, E., \& Krueger, D. 1997, in Astrophysical Implications of the Laboratory Study of Presolar Materials, ed. T.J. Bernatowicz \& E. Zinner, AIP Conf. Proc. 402, 425 - 450

Steidel, C.C., et al. 1999, ApJ, 519, 1

Tielens, A.G.G.M. 1998, ApJ, 499, 267

Verter, F., Magnani, L., Dwek, E., \& Rickard, L.J. 2000, ApJ, 498, 831

Witt, A.N. 2000, JGR, 105, 10299

Witt, A.N., \& Gordon, K.D. 2000, ApJ, 528, 799

Witt, A.N., Gordon, K.D., \& Furton, D.G. 1998, ApJ, 501, L111

Zaritsky, D. 1994, AJ, 108, 1619 